\title{
Solution Modeling in System Coordination of Objectives and Resourcing Linear Business Processes
}

\author{
Valeriy Kanev \\ Mathematical Modeling of Business Processes Department, \\ Siberian State University of Telecommunications and Information Science, Novosibirsk \\ E-mail:kanev@ngs.ru \\ Received 20 February 2017; accepted 18 April 2017
}

\begin{abstract}
The paper deals with the analytical approach to reaching compromises in coordinating objectives and resourcing business processes, algorithms and software for generating strategies - compromises for situational analysis. Unlike traditional schemes it is suggested using a vector-mode display to analytically manage both resources in a risk-tolerant way and signaling event occurrence dates in a technologically practicable schedule of a net model. Model-methodical support of linear business processes realization programs can be used for the algorithmic control of objectives coordination and resourcing when controlling program realization of linear engineering, e.g. highway and oil-and-gas pipeline engineering, railway and power lines engineering, etc., i.e. engineering of technologically one-type objects linearly allocated in space. The experimental calculations based on actual construction programs confirmed the model construction adequacy, practical usefulness of the modeling results and modeling solutions in the system coordination of objectives and resourcing linear business processes.
\end{abstract}

Keywords: modelling, coordination, net model, compromises, business process, vector-mode display.

JEL Classification: C61, M10, M21.

Conference topic: Business Logistic Processes.

\section{Introduction}

Mathematical representation of developing a set of events based on a long-life network model under limited resources and scheduled time of the deadline issues the challenge of coordinating these signaling dates and funding required. In case of discoordination different risks in achieving business goals arise (Merkevicius et al. 2015; Stádník et al. 2016). Their character lies in various behavior modes and response mechanisms of market agents to any particular preferences: tightening or liberalizing the dates of signaling event occurrence and options of expectative dynamics of the resources consumed.

Risk indicators in reaching objectives in the system involved depend on the "singularity" and network constraints describing organizational technologic element links of business processes giving rise to domain walls of minimum obligatory and maximum technologically allowed funding (Burinskiene, Pipiriene 2014; Davidaviciene 2008). Knowing these domain walls provides best practices to quickly monitor and assess the balance and consistency of the resource supply plans in compliance with the scheduled time of business process realization as a whole (Burinskiene 2015; Davidaviciene, Meidute 2012). A risk measure is traditionally built on the basis of the geometrical probability definition (Janeliuniene, Davidaviciene 2012). Kanev (2014) assuming domain walls of minimum required and maximum available funding (Fig. 1) as a set of all elementary outcomes.

\section{Previous research}

The most suitable economic-mathematical tools for solving tasks of coordinating objectives and resourcing linear business processes are classical models and modified Zukhovsstskii and Radchik (1965). Moder and Phillips (1966) network models clearly describing technological and time laws in discoordinating and isolating risk factors in achieving final and intermediate goals of deploying linear business processes (Aleshina 2004).

(C) 2017 V. Kanev. Published by VGTU Press. This is an open-access article distributed under the terms of the Creative Commons Attribution (CC BY 4.0) License, which permits unrestricted use, distribution, and reproduction in any medium, provided the original author and source are credited. 
At the same time solves the problem of integration of different network models of systems in the control circuits (Rybalsky 1979; Rykunov 2000; Prykin 1991; Pavlovsky 1979). Management of large-scale building complexes on the basis of network models were made from the perspective of system analysis and control theory (Larin 1994).

The task of coordinating objectives and its well-balanced resourcing risk is aggregated as follows (Aleshina 2004; Rybalsky 1979). The circuit-free oriented graph $\mathrm{G}$ without end vertices with jobs is set $i j \in G$.

For every network graph the job performance time $\tau_{i j} \geq 0$, a resource consumed $l_{i j} \in L$ and annual dynamics of its utilization according to the schedule date $[0, \mathrm{~T}]$ when doing job at the time zero $b_{i j}(0)$ have been defined.

On the network graph it is critical to specify the following scheduled job $P=\left\{t_{i j} \mid i j \in G\right\}$, where $t_{i j}-$ starting time of job performance $\mathrm{ij}$, wherein the dynamics of resource employment $R(P)=\left\{R_{l t}(P) \mid t=1, \ldots, T ; l \in L\right\}$ deviate minimally from the prescribed dynamics $K=\left\{K_{l t} \mid t=1, \ldots, T ; l \in L\right\} \sum_{l \in L} \sum_{t=1}^{T}\left(R_{l t}(P)-K_{l t}\right)^{2} \rightarrow \min _{P=\left\{t_{i j}\right\}}$ under normal limitations at the starting point of performance, event time and scheduled control event time.

In this case, scheduled event time and suggested planned resource dynamics $K$ are defined model-independently by experts. The balance requirement should be imposed to dynamics, because unbalanced resources development either hinders investment process from development (at high peaks of resources employment), or supposes irrational use of available production capacity (at sharp fall of utilization capacity).

The problem was solved as a rule by man-machine interaction, supposing that technologically available employment of each resource was initially determined under scheduled dates given. The approach proposed in the paper results is free of «interactive subjectivity» and aimed at analytical algorithm presentation of the problem considered including its multiresource character.

For methodological generality, it should be noticed that the attempts (Kanev 1981, 2009) of a non-classical network model for solving the issues in question have already been undertaken. Objectives coordination and resourcing process have been described with the system of finite difference equations with piecewise continuous control - time functions and a definite set structure $U(t)$ has been assigned about these functions. Given that starting time of jobs has been described with the Heaviside function. Because of solving this task based on Pontryagin's maximum principle optimal control has been chosen, i.e. business process realization time has been minimized with due account for resource and technological (network) restrictions to program implementation (Bouleimen, Lecocq 2003).

The main peculiar feature and advantage of this approach consists in solving an inverse task - variable intensities. It eliminates the need to fix the duration of job performance on the network model thus greatly facilitating the information support of tasks in the network statement. Constructing a control model based on the discrete principle of maximum absolutely differs from the approach using a network planning unit or any other mathematical programming method in the fact that all variables are unequal both in the model record itself and in the solution algorithm and result interpretation. Deliberate division of the whole space of variables into a subspace of program states and that of control actions makes meaningful discussions of program realization on the monitoring and control stage more natural. However, these modifications have not provided any analytical relations with the informative aspect obtained in the Results.

\section{Methodology}

The research methodology uses evidence-based approach that for all available job schedules, network model measures the composed functions appropriately designed for network capacity belong to the enclosed domain, and a set of constraints describing this domain is determined. The distinctive feature of these constraints is that they do not depend on the schedule date of business process realization because the extreme left schedule is the same under different schedule dates (Kolish, Hartmann 2006). Moreover, using these relations one can know what adjustments should be made in the resources supply plan, if it is not coordinated with the schedule date of the business process realization.

In one-resource model the business process realization is described by the network graph $G$ on the time interval $[O, T]$ (circuit-free and without end vertices) with jobs $i j \in G=G_{1} \cup G_{2}$ where $G_{1}$ - a set of jobs requiring a resource to be performed, $G_{2}$ - a set of $i j \notin G_{1}$. Job time $\tau_{i j}(i j \in G)$ and dynamics of resource utilization at the time zero of their performance initiation $b_{i j}(O)\left(i j \in G_{1}\right)$ are given at the edges of a graph. Job time and resource utilization capacity by period performance are not supposed to depend on the time calendar intervals, herewith jobs are performed without interruption within them, model variables are the following: $\mathrm{P}=\left\{P_{i j} \mid i j \in G\right\}-$ a schedule time of a net graph $G$, stating for all $i j \in G$ their performance initiation time $P_{i j}$, so that $b_{i j}\left(P_{i j}\right)=\left\{b_{i j}^{n}\left(P_{i j}\right) \mid n=\overline{1, T}\right\} \quad\left(i j \in G_{1}\right)$ - are vectors characterizing dynamics of resource utilization by planning periods in the course of jobs $i j \in G_{1}$ at their performance 
initiation at points of calendar time $P_{i j}$. Then $X(P)=\sum_{i j \in G_{1}} b_{i j}\left(P_{i j}\right)$ - a vector characterizing dynamics of resource aggregated utilization at scheduled job performance $P$.

\section{Results}

Mapping the schedule $P$ by a resource development vector $X(P)$ one can take advantage of a square matrix inversion A of size $T$, having the following structure:

$$
\boldsymbol{A}=\left[\begin{array}{cc}
\boldsymbol{O}_{1=(\boldsymbol{T}-1)} & 1 \\
\varepsilon_{(\boldsymbol{T}-1) \boldsymbol{x}(\boldsymbol{T}-1)} & \boldsymbol{O}_{(\boldsymbol{T}-1) \boldsymbol{x} 1}
\end{array}\right], \text { where } \varepsilon, \boldsymbol{O}-\text { identity and zero matrices respectively. }
$$

When using these matrices and parameters

$$
\begin{aligned}
& \lambda_{i j}=\left\{\begin{array}{c}
1-\tau_{i j}+\left[\tau_{i j}\right], \quad \text { if } \tau_{i j}>\left[\tau_{i j}\right] \\
\boldsymbol{O}, \quad \text { if } \tau_{i j}=\left[\tau_{i j}\right] \quad\left(\boldsymbol{i j} \in \boldsymbol{G}_{1}\right)
\end{array}\right\}, \quad \text { a network model is put in the logical way: } \\
& P_{i j}=t_{i j}+\left(1-\alpha_{i j}\right) \Delta_{i j}+\alpha_{i j} \lambda_{i j}\left(i j \in G_{1}\right) ; \\
& t_{i j} \geq O, \quad \text { integer }\left(i j \in G_{1}\right) \text {; } \\
& \Delta_{i j}=\left\{\begin{array}{ll}
O, & \left(i j \in G_{1}\right) \\
1 & \left(i j \in G_{1}\right)
\end{array}\right\} \\
& O \leq \alpha_{i j} \leq 1 \quad\left(i j \in G_{1}\right) .
\end{aligned}
$$

Points of calendar time $P_{i j}$ of job performance initiation $i j \in G_{1}$ are given as scalar functions of arguments $\mathbf{t}_{i j}, \alpha_{i j}, \Delta_{i j}$, being the parameters mapped $P_{i j}$ in $b_{i j}\left(P_{i j}\right)$ under constraints $(2)-(4)$;

$$
P_{i j}+\tau_{i j} \leq P_{j k} \quad(i j, i k \in G) .
$$

Network constraints at points of job performance initiation:

$$
P_{i n}+\tau_{i N} \leq T \quad(i N \in G) .
$$

The initiation point of a top event $N$ of graph $G$ does not exceed the schedule date duration:

$$
P_{i j}+\tau_{i j} \leq S_{j} \quad(j \in Z \subset G, i j \in G) .
$$

For a set of milestones $Z$ their initiation points do not exceed schedule dates $S_{j}$;

$$
X(P)=\sum_{i j \in G_{1}}\left(\left(1-\alpha_{i j}\right) A^{t_{i j}+\Delta_{i j}} b_{i j}(O)+\alpha_{i j} A^{t_{i j}} b_{i j}\left(\lambda_{i j}\right)\right) .
$$

The aggregated dynamics of resource utilization $X(P)$ is determined by scheduled jobs $P$ of a network graph $G$.

At the first-stage control coordination of long-term the true progress of jobs is required to be assessed. It includes: $S=\left\{S_{j} \mid j \in Z \subset G\right\}-$ a schedule date of step-by-step business process realization (objectives): $K=\left\{K_{n} \mid n=\overline{1, T}\right\}-$ a plan dynamics of investments. The formal objective is to analyze the system solvability (I) - (8) under constraints:

$$
X_{n}(P)=K_{n}^{H} \quad(n=\overline{1, T}) .
$$

In case investments are considered to be non-stock resources:

$$
\sum_{n=1}^{m} x_{n}(p) \leqslant \sum_{n=1}^{m} K_{n}^{c}(m=\overline{1, T})
$$

If it is supposed to stock up investments in time. 
It is suggested to start analyzing with calculations of late available start of job performance initiation $t_{i j}^{n H}(i j \in G)$ under constraints (5)-(7) and a delay detection in the program realization deadline:

$G_{3}=\left\{i j \in G_{1} / t_{i j}^{n H}<0\right\}-$ a set of jobs, for which a lag is admitted ${ }^{1}$;

$3_{i j}=\left\{\begin{array}{c}0\left(i j \notin G_{3}\right) \\ \min \left(-t_{i j}^{n \mu}, \tau_{i j}\right)\left(i j \in G_{3}\right)\end{array}\right\}-$ time delays in job performance dates;

$3_{i j}^{H}=\max _{k i \in G}\left(3_{k i}^{H}+3_{k i}\right)(i j \in G)-$ time delays to the point of job performance start;

$3_{i j}^{o}=3_{i j}^{H}+3_{i j}(i j \in G)-$ time delays to the point of job completion;

$Q_{i j}=\sum_{n=1}^{\left[3_{i j}\right]} b_{i j}^{n}+b_{i j}^{\left[3_{i j}\right]+1}(O) \times\left(3_{i j}-\left[3_{i j}\right]\right)\left(i j \in G_{3}\right)-$ volume of underinvestments for jobs;

$Q=\sum_{i j \in G_{3}} Q_{i j}-$ a total volume of underutilized means.

As alternative solutions of delays the reduction job intensification (critical path reduction task), business process reengineering either job organization flow change (graph topology variability) or deadlines extension are suggested (Qi et al. 2014). Revealed deviation removed and model parameters appropriately updated, coordination of planned investment behavior with a schedule time of business process strategic parameters realization at a set of available schedules is analyzed.

This analysis is supposed to start with dynamics prediction of investment utilization from the extreme left $X\left(P^{\pi}\right)$ and extreme right $X\left(P^{\mathrm{n}}\right)$ job schedule. It is shown that for the model (1-8) the following is correct $x(P) \subset D=D^{n} \cap D^{n}$, where

$$
\begin{aligned}
& D^{n}=\left\{Y \in E^{T} / \sum_{n=1}^{m} Y_{n} \leq \sum_{n=1}^{m} X_{n}\left(P^{n}\right)(m=\overline{1, T})\right\}, \\
& D^{n}=\left\{Y \in E^{T} / \sum_{n=1}^{m} Y_{n} \leq \sum_{n=1}^{m} X_{n}\left(P^{n}\right)(m=\overline{1, T})\right\} .
\end{aligned}
$$

Based on the above the regulating restrictions system for investments plan admissibility and algorithmically simple test procedures of its coordination with the schedule time of the implementation is set:

$$
\sum_{n=1}^{m} X_{n}\left(P^{n}\right) \geq \sum_{n=1}^{m} K_{n}^{\mu} \geq X_{n}\left(P^{n}\right)(m=\overline{1, T})
$$

The requirement for a plan feasibility in case of a non-stock resource is:

$$
\sum_{n=1}^{m} K_{n}^{c} \geq \sum_{n=1}^{m} X_{n}\left(P^{n}\right)(m=\overline{1, T})
$$

A necessary and sufficient condition for a plan admissibility in case of a stock resource is:

$$
\begin{gathered}
\tilde{K}_{1}^{H}=K_{1}^{H}-\max \left(O, K_{1}^{H}-X_{1}\left(P^{n}\right)\right) ; \\
\widetilde{K}_{1}^{H}=K_{1}^{H}-\max \left(O, K_{n}^{H}+\sum_{m=1}^{n-1} \tilde{K}_{m}^{H}-\sum_{m=1}^{n} X_{m}\left(P^{n}\right)\right)(n=\overline{2, T}) .
\end{gathered}
$$

The maximum utilization dynamics of a non-stock resource as intended is as follows:

$$
\begin{aligned}
& H_{n}^{c}=\max \left(O, \sum_{m=1}^{n}\left(X_{m}\left(P^{n}\right)-K_{m}^{c}\right)\right)(n=\overline{1, T}) ; \\
& H_{n}^{H}=\max \left(O, \sum_{m=1}^{n}\left(X_{m}\left(P^{n}\right)-\tilde{K}_{m}^{H}\right)\right)(n=\overline{1, T}) .
\end{aligned}
$$




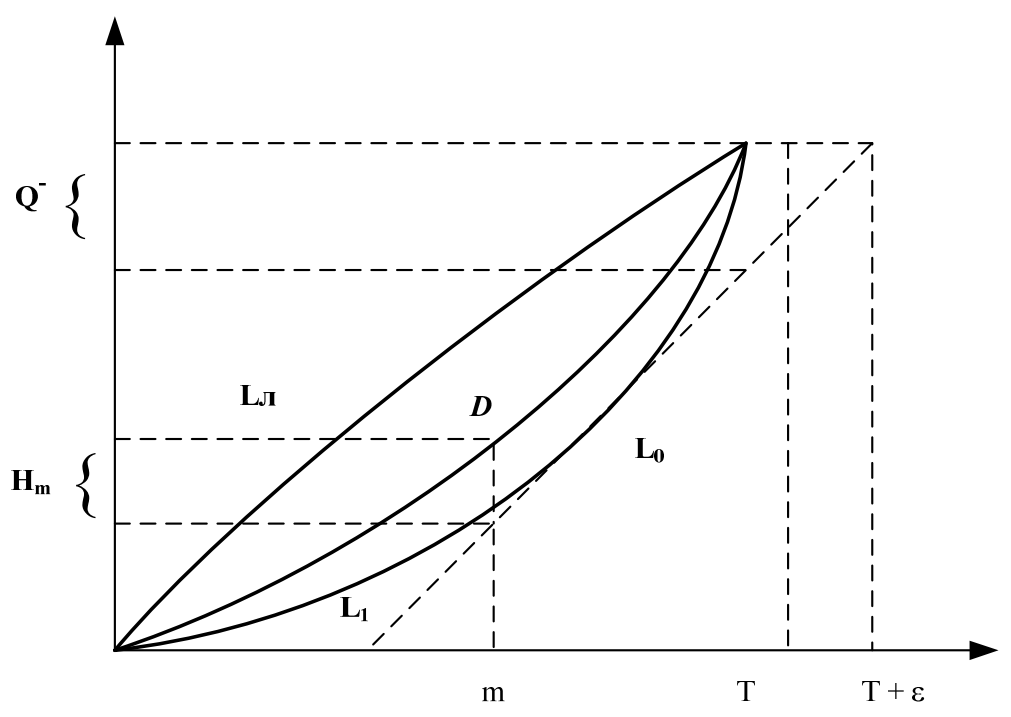

Fig. 1. Diagram of analysis for the technologically feasible investment utilization domain

The amount of the minimum required increase of a resource volume for $n$ first measures of the schedule date $[0, \mathrm{~T}]$ for a program realization in a schedule time

$$
\varepsilon \geq l-1 .
$$

The lower bound of the minimum possible total delay for all schedule dates due to a lack of investments, where $l-$ a minimum possible integer, for which the following inequality is true:

$$
\begin{gathered}
\sum_{n=1}^{m-l} X_{n}\left(P^{n}\right) \leq \sum_{n=1}^{m} \tilde{K}_{n}^{H} \quad(m=l+\overline{1, T}) \\
\sum_{n=1}^{m-l} X_{n}\left(P^{n}\right) \leq \sum^{m} K_{n}^{c} \quad(m=l+\overline{1, T}) \\
Q^{-} \geq \sum_{n=T-l+1}^{T} X_{n}\left(P^{n}\right) .
\end{gathered}
$$

The minimal volume of fund underutilization on the interval $[0, T]$ due to a time delay $\varepsilon_{m}$

$$
\tilde{N} \geq \max _{m=1, T} \frac{\sum_{n=1}^{m} X_{n}\left(P^{n}\right)}{m} .
$$

The lower bound of a technologically insuperable vertex of the resource development on the interval $[0, T]$.

\section{Discussion}

Graphically basic relationships are shown on Figure 1, where $L_{n}, L_{n}$-accumulation curves of investment utilization from the extreme left and extreme right job schedule, $L_{0}$ - a planned dynamics of investments.

The alternative elimination of the unbalance found is:

- a planned investment dynamics change (II) - (I6), (I9),

- extending deadlines of the whole business process realization (I7),

- a job scope reduction (I8) or its intensification (shortening of the critical path or a graph topology variation).

In the latter the domain $D$ must be enlarged in such a way for the planned dynamics curve $L_{0}$ to be inside it (Vanhoucke et al. 2001).

At the next test stage it is supposed to freeze the domain of feasible search for a next-scheduled current plan $\Pi=\left\{\Pi_{i j} / i j \in G_{1}\right\}$ investment utilization for program jobs: $b_{i j}^{1}\left(t_{i j}^{P H}\right) \geq \Pi_{i j} \geq b_{i j}^{1}\left(t_{i j}^{n H}\right) \quad\left(i j \in G_{1}\right)-$ a planned volume of the investment utilization in this year job $i j \in G_{1}$ cannot exceed the utilization volume at its early start $t_{i j}^{P H}$ cannot 
be lower than the utilization volume at late allowable date $t_{i j}^{n \mu} ; \sum_{i j \in G_{1}} \Pi_{i j}=K_{1}-$ a cumulative volume of the planned utilization of investments equals their year limit.

To analyze the adopted plan and its adjustment it is suggested to bring in a model (I)-(8) the following constraints:

$$
P_{i j}=1-\Pi_{i j} / b_{i j}^{1}(0) \quad\left(i j \in G_{1}\right)
$$

and to start job performance according to the adopted plan.

The model (I)-(I0), (20) is analyzed on the basis of the above scheme that assesses the performance consequences of the adopted current plan.

In a multiresource network model the investments are differentiated on business process objects and the constraint (8) in the model (I) - (I0) is modified as follows:

$$
X^{m}(P)=\sum_{i j \in G_{1}}\left(\left(1-\alpha_{i j}\right) A^{t_{i j}+\Delta_{i j}} b_{i j}^{m}(0)+\alpha_{i j} A^{t_{i j}} b_{i j}^{m}\left(\lambda_{i j}\right)\right) \quad(m=\overline{1, M}),
$$

where:

$m$ - an index of the resource utilized and $M$ - the amount; $b_{i j}^{m}\left(P_{i j}\right)$ - a $T$-measured vector of $m$ - resource utilization in the job $i j \in G_{1}$ at its start at the time point $P_{i j} X^{m}(P)$ - a sum vector of utilization of the $m$ - a resource when performing all jobs $i j \in G$ on the schedule $P$.

The investment plan in a multiresource task is as follows:

$$
K_{m}=\left\{K_{m n} / n=\overline{1, T}\right\} \quad(m=\overline{1, M}) .
$$

The test procedures in this case can be performed on the above scheme either for a fixed allocation of investments or with a free distribution of investments between business process objects.

A few more considerations on the separation of procedures and functions for monitoring the coherence of targets and resource security. The implementation of operational management of the progress of large-scale linear construction requires that the level of senior management be concentrated at the level of centralized control over the fulfillment of final and intermediate program-target tasks. This leads to a vertical disaggregation of the monitoring function by the levels of program management to the separation of strategic (general) control from tactical (operational) in the centralization of the first and decentralization of the second. In contrast to tactical control over the implementation of current planning decisions, strategic control should be aimed at solving problems of effective use of resources in the future to achieve the ultimate program goals and should concentrate attention of the top management on the implementation of program-target tasks, rather than on obtaining current "momentary" results.

It has been repeatedly established (Kanev 1981) that in practice these aspects of control are often sacrificed to the tasks of operational management. The inaccuracy of certain planning decisions taken by the program bodies in their orientation towards obtaining current results does not often lead to a breakdown in the implementation of target tasks. This reduces the software development impact and leads to significant losses.

In these conditions, a systematic centralized control is needed not only for implementation, but also for the development of plans. The essence of this control should not consist in fixing the inadequacy of the plan to the ultimate program objectives, but in preventing it by timely forming the requirements that the plan must objectively satisfy.

When implementing long-term programs, the inadequacy of centrally-made decisions to ultimate goals is manifested, on the one hand, in the imbalance of certain sections of the Perspective plan, and on the other hand, in the inconsistency of current and future planning assignments. Ensuring the balance and consistency of plans in the process of implementing the program is difficult due to a constant change in its status and the possibility of emergence of situations where the originally adopted directive cannot be fulfilled. Therefore, the centralized control is offered consistently in two stages:

- control of the implementation of the long-term plan and its adequacy to the actual state of the program;

- monitoring the consistency of current and future planning targets.

That is why we hope that the development of a model algorithmic apparatus for the implementation of such control can be performed on the basis of the decomposition approach to modeling the process of program management. This requires a system of models of at least three types:

- accounting,

- control,

- planning.

The accounting model should be used to analyze the accumulated statistical information and to reveal the patterns inherent in the controlled object.

Planning model - to optimize planning decisions. 
The model of control - for the formation of endogenous constraints (hereinafter area D, Fig. 1), which should be met by allowable plans, and to assess the consistency of these constraints with exogenously specified goals.

It is quite natural that in order to control the implementation of a long-term construction program, it is advisable to use the network model in the simulation mode. Procedures for analyzing such a model should be based on the set of its admissible schedules for given periods of occurrence of control events, since the implementation of the same fixed directive detailed schedule for the implementation of the long-term program, on the one hand, is unrealistic, and with the other, Maneuvering resources, which reduces the flexibility of management and reliability of the implementation of the ultimate program objectives (Bouleimen, Lecocq 2003).

We have worked out the basic scheme of automatic control of the program with the use of an imitation control system, which includes blocks for modifying the state of the program, displaying the accepted hypotheses by the network model, diagnosing errors and evaluating the planned decisions. In this case, as shown above for monitoring, you can use both a single resource and a multi-resource network model, in which capital investments can relate to a stored or non-storable type (Neumann, Zimmermann 2000).

To check the performance of model-methodological support several experimental calculation cycles were carried out. These calculations were carried out on a multi-resource network model of a modular structure. The model contains more than 2 million jobs.

A peculiar feature of the calculations is their direct practical focus on two levels of management: management of investment resources and direct management of the owners of the business processes.

The first series of experimental calculation was performed at the initial stage of a fifteen-year investment cycle. As a result of the calculations, it was found that the overall deadline starting complex of all large-scale project was directly predetermined by a number of specific business processes, having a "cork" character for a linear structure. It should be paid the utmost attention. Envisaged in the draft the construction organization would provide the realization of the entire program at substantial intensification of work (1.4 times) and increasing the volume of investments by $17 \%$ from the initial plan.

The next series of calculations was performed at the regular time of monitoring. Calculations confirmed the possibility to meet the planned deadlines of the project, but to meet several intermediate deadlines requires a fairly significant increase in funding of specific business processes. Thus, the results obtained, on the one hand, have shown the inconsistency of a design options-term plan with the actual state and identified the need to adjust it. On the other hand, they have shown the groundlessness of the rational spending hypothesis adopted at the first stage of calculations.

The next series of calculations has been performed because of the new government decrees on changing the investment process structure and volumes. Calculations have defined the area of technologically necessary funding (area D, Fig. 1) for meeting deadlines and policy developments. Further, in a monitoring mode at each stage the calculations were carried out to assess the feasibility of implementation of the approved plan of business processes with the diagnosis of critical and sub-critical range of jobs with the risk assessment of the exit from the domain D and the parameters of necessary intensification of separate business processes. All these results have shown high efficiency of the developed model-methodological support. Calculations and detailed scenarios including a quantitative assessment of the simulation results one can see in the following sources (Kanev 1981; Kanev, Shevtsova 2015, 2014).

\section{Limitations}

All fundamental results have been obtained analyzing linear business processes. However, linearity itself was particularly a peculiarity simplifying the job with a network model. Standardized modules - a network graph of the elementary business process (interstation spans when railway engineering) have been replicated in a special way having a connection in the spaced generic linear net structure (e.g., when modeling a gas pipe line construction - the whole main pipe). The obtained results on the marked space analytics of minimum technologically required and maximum technologically possible funding (at fixed network topology) can be used without any negative impact for nonlinear business processes.

It should be noted that in the scientific and methodical works and educational materials of recent years can be traced quite clearly by the authors (Phillip, Garcia-Diaz 1984; Project Management Institute 2008; Anshin, Ilyina 2013) position on the need to allow communication of the analytical resource vector network model with the parameters of the possible and feasible schedules.

\section{Conclusions}

Summing it up, at least three conclusions can be made. First, model-methodical support of linear business processes realization programs can be used for the algorithmic control of objectives coordination and resourcing when controlling program realization of linear engineering, e.g. highway and oil-and-gas pipeline engineering, railway and power lines engineering, etc., i.e. engineering of technologically one-type objects linearly allocated in space.

Secondly, using specialized software and algorithms support one can fill in the input documents, find errors в in the given data, analyze aggregated indicators of job progress, simulate the workflow organization with a network 
model, outline deviations in construction periods, causes and ways of job intensification, and also make multiple-path calculations of modeling balances when controlling planned dynamics coordination of investments under the scheduled time of putting an object into action. All calculations can be made in a cross-section of the whole business process and an aggregated package of jobs for various kinds of their performance (optimistic, pessimistic, expected) both taking into account the adopted current plan and ignoring it.

Thirdly, the experimental calculations based on actual construction programs confirmed the model construction adequacy, practical usefulness of the modeling results and modeling solutions in the system coordination of objectives and resourcing linear business processes.

\section{References}

Aleshina, S. 2004. Science netting, Company's secret 47(86): 51-55.

Anshin, V.; Ilyina, O. 2013. Project management. Fundamental Course. Textbook. Moskow: Higher School of Economics (State University) Ltd.

Bouleimen, K.; Lecocq, H. 2003. A new efficient simulated annealing algorithm for the resource-constrained project scheduling promlem and its multiple modes version, European Journal of Operational Research 149: 268-281. https://doi.org/10.1016/S0377-2217(02)00761-0

Burinskienè, A. 2015. Optimising forklift activities in wide-aisle reference warehouse, International Journal of Simulation Modelling 14(4): 621-632. https://doi.org/10.2507/IJSIMM14(4)5.312

Burinskienè, A.; Pipirienè, V. 2014. Technological innovations and technology achievement, in The $8^{\text {th }}$ International Scientific Conference "Business and Management 2014", 15-16 May 2014, Vilnius, Lithuania.

Davidavičienė, V.; Meidute, I. 2012. Supply chain models in the Lithuanian e-commerce, in Contemporary Issues in Business, Management and Education'2012, 15 November 2012, Vilnius, Lithuania.

Davidavičienè, V. 2008. Change management decisions in the information age, Journal of Business Economics and Management 9(4): 299-307. https://doi.org/10.3846/1611-1699.2008.9.299-307

Herroelen, W.; Leus, R. 2005. Project scheduling under uncertainty: survey and research potentials, European Journal of Operational Research 165(2): 289-306. https://doi.org/10.1016/j.ejor.2004.04.002

Janeliūnienè, R.; Davidavičienè, V. 2012. Information technology risks identification: peculiarities of small and medium sized enterprises, in Contemporary Issues in Business, Management and Education'2012, 15 November 2012, Vilnius, Lithuania.

Kanev, V. S. (Ed.). 1981. Optimization program line construction. Novosibirsk: Science, Siberian Branch.

Kanev, V. S. 2009. Methodological and methodical questions of construction of system models of regional info-communication complex, Vestnik SibGUTI No. 1: 3-25.

Kanev, V. S. 2014. System risk management methods, models and their implementations in telecommunications, Scientific Bulletin of National Mining University 4: 93-100.

Kanev, V. S.; Shevtsova, Y. V. 2015. Fundamentals of modeling and operational risk management in e-commerce and telecommunications. Moskow: Hotline-Telecom.

Kanev, V.; Shevtsova, Y. V. 2014. Posterior modeking of operational losses, Scientific Bulletin of National Mining University 4: $133-138$.

Kolish, R.; Hartmann, S. 2006. Experimental investigation of heuristics for resource-constrained project scheduling: an update, European Journal of Operational Research 174(1): 23-37. https://doi.org/10.1016/j.ejor.2005.01.065

Larin, A. A. 1994. Theoretical bases of management. G. 1.: The processes and management systems. Moskow: PBCH.

Merkevičius, J.; Davidavičienè, V.; Raudeliūnienè, J.; Buleca, J. 2015. Virtual organization: specifics of creation of personnel management system, E\&M Economics and Management 18(4): 200-211.

Moder, J.; S. Phillips, S. 1966. Network planning method in the works, from English. Rome, Moskow - Lendingrad.

Neumann, K.; Zimmermann, J. 2000. Procedures for resource levelling and net present value problems in project scheduling with general temporal and resource constraints, European Journal of Operational Research 127: 425-443.

Pavlovsky, Y. N. 1979. Decomposition models of control systems. Moskow: Science.

Phillips, D.; Garcia-Diaz, A. 1984. Methods of network analysis: from English. Moskow: Mir.

Project Management Institute. 2008. Project management manual (Manual PMBOK), 4-th publ. Four Campus Boulevard, Newtown Square, PA 19073-3299 USA / USA ANSI/PMI 99-001-2008.

Prykin, B. V. 1991. Management Basics. Industrial and building systems: a textbook for high schools. Moskow: Stroyizdat.

Qi, J.; Guo, B.; Lei, H.; Zhang, T. 2014. Solving resource availability cost problem in project scheduling by pseudo particle swarm optimization, Journal of Systems Engineering and Electronics 25(1): 69-76.

Rybalsky, V. I. 1979. Automated building management system. Kiev: Kiev High school Ltd.

Rykunov, V. I. 2000. Management Basics: Monograph. Moskow: Izograf.

Stádník, B.; Raudeliūnienè, J.; Davidavičienė, V. 2016. Fourier analysis for stock price forecasting: assumption and evidence, Journal of Business Economics and Management 17(3): 365-380.

Vanhoucke, M.; Demeulemeester, E.; Herroelen, W. 2001. On maximizing the net present value of a project under renewable resource constraints, Management Science 47(8): 1113-1121.

Zukhovsstskii, S. I.; Radchik, I. A. 1965. Mathematical methods of network planning. Moskow. 